\title{
Retour d'expérience sur la crise Covid-19 dans une maternité française de type 3
}

\author{
Feedback on COVID-19 Outbreak in a French Tertiary Maternity
}

\author{
F. Vial $\cdot$ L. Mortier $\cdot$ J. Rouche $\cdot$ C. Mézan de Malartic $\cdot$ D. Herbain $\cdot$ E. Gauchotte $\cdot$ H. Bouaziz $\cdot$ J.-M. Hascoet $\cdot$ \\ O. Morel
}

Reçu le 5 avril 2020 ; accepté le 22 août 2020

(C) SFMU et Lavoisier SAS 2020

Résumé L'arrivée brutale de l'épidémie sanitaire liée au SARS-CoV-2 dans la région Grand Est a conduit les services de soins de cette région à s'adapter rapidement à la situation. Le Plan blanc a été déployé dans les hôpitaux de Strasbourg et Nancy dès le 13 mars 2020 afin de faire face à un afflux massif de patients. Face à cette urgence sanitaire d'un type nouveau, la maternité de Nancy s'est réorganisée forte de l'expérience des services strasbourgeois rapidement mis en tension afin d'assurer l'accueil et de dispenser les meilleurs soins possible aux parturientes dans un environnement limitant au maximum leur risque de contamination. Du fait du haut risque de transmission virale, un suivi anténatal par téléconsultations et visites à domicile par le réseau de ville (sages-femmes libérales, généralistes) a été organisé pour les grossesses à bas risque. Sur site, un triage des admissions a rapidement été mis en place ainsi que la création d'un circuit spécifique dédié aux patientes à risque ou confirmées porteuses du SARS-CoV-2. Des procédures de prise en charge gynéco-obstétricales, anesthésiques et pédiatriques

F. Vial $(\bowtie) \cdot$ L. Mortier · D. Herbain · H. Bouaziz

Service d'anesthésie réanimation, maternité universitaire, pôle d'anesthésie, centre hospitalier régional et universitaire (CHRU) de Nancy,

10, rue du Docteur-Heydenreich, F-54000 Nancy, France

e-mail : f.vial@chru-nancy.fr

J. Rouche $\cdot$ C. Mézan de Malartic $\cdot$ E. Gauchotte $\cdot$ O. Morel Pôle de gynécologie et obstétrique,

maternité universitaire, CHRU de Nancy,

10, rue du Docteur-Heydenreich, F-54000 Nancy, France

\section{J.-M. Hascoet}

Service de néonatologie, maternité universitaire, pôle enfants-néonatologie, CHRU de Nancy,

10, rue du Docteur-Heydenreich F-54000 Nancy, France

H. Bouaziz · J.-M. Hascoet · O. Morel

Faculté de médecine, université de Lorraine,

9, avenue de la Forêt-de-Haye, F-54547 Vandœuvre-lès-Nancy,

France ont été rédigées et révisées par les différentes équipes au fur et à mesure de l'évolution des connaissances et des recommandations émises par les sociétés savantes. L'objectif de cet article est de détailler l'ensemble de l'organisation mise en place, de décrire les difficultés de fonctionnement rencontrées lors du pic de l'épidémie et d'analyser les points d'amélioration mis en évidence.

Mots clés Covid-19 Coronavirus · Maternité $\cdot$ Retour d'expérience $\cdot$ Organisation des soins

\begin{abstract}
The sudden arrival of the SARS-CoV-2 outbreak conducted healthcare departments to rapidly adapt their organizations. The White Plan has been activated in Strasbourg and Nancy the March 13, 2020 to deal with a massive influx of patients. Due to this new kind of sanitary emergency, build on Strasbourg's experience, the University Maternity of Nancy has been reorganized in order to propose the best care conditions for women in an environment limiting the risk of contamination. In view of the high risk of viral transmission, antenatal follow-up by teleconsultations and home visits provided by independent midwives and general practitioners was promoted for low-risk pregnancies. A pre-admission triage system and a specific circuit for patients suspected or infected with SARS-CoV-2 have been created. Specific procedures of care in anesthesiology, obstetrics and pediatrics have been drawn up and regularly revised according to national recommendations and literature. The aim of the article is to describe our health care organization, to highlight operational difficulties and points of improvement identified.
\end{abstract}

Keywords COVID-19 - Coronavirus - Maternity · Feedback $\cdot$ Healthcare strategy 


\section{Introduction}

L'arrivée en France du SARS-CoV-2 a conduit les établissements hospitaliers de la région Grand Est à s'adapter en quelques jours à une crise sanitaire sans précédent. Le 19 mars 2020, la région Grand Est est devenue le deuxième lieu de circulation intense du virus après l'Île-de-France. Les villes de Mulhouse, Colmar, Strasbourg puis Metz ont vu rapidement leurs services de réanimations médicales, chirurgicales et polyvalentes saturés et leurs effectifs soignants diminués du fait d'un absentéisme marqué lié au virus particulièrement contagieux. Les projections épidémiologiques réalisées en mars 2020, annonçant une explosion du nombre de cas d'infections à SARS-CoV-2 sur le territoire de santé du centre hospitalier régional et universitaire (CHRU) de Nancy avec un décalage d'environ une semaine par rapport à l'Alsace, ont conduit à une adaptation rapide des services de soins de l'établissement afin de faire face à la crise sanitaire. Annonce du Plan blanc, fermeture des blocs opératoires, report des consultations non urgentes se sont alors imposés.

Seul établissement de type 3 de la subdivision lorraine, la maternité régionale de Nancy, forte de l'appui des maternités strasbourgeoises et mulhousiennes sévèrement impactées dès le début de l'arrivée du virus sur le territoire français, a mis à profit cette semaine d'avance sur l'arrivée du pic épidémique pour réorganiser dès le 15 mars 2020 ses activités. Les objectifs étaient doubles :

- assurer la continuité des soins tout en garantissant un confinement optimal des patientes et des soignants ;

- mettre en place une organisation adaptée à l'accueil de patientes suspectées infectées ou infectées par le SARSCoV-2 (dépistage et traitement) dans le cadre spécifique des activités de gynécologie-obstétrique.

L'objectif ici est de décrire l'organisation locale mise en place pour faire face à la pandémie afin de servir de support à d'autres maternités et de rapporter les difficultés rencontrées. En effet, à ce jour, aucune recommandation n'existe quant à l'adaptation des moyens techniques et humains à mettre en œuvre dans de telles situations en gynécologie-obstétrique.

\section{Réorganisations mises en ouvre en amont de la survenue du pic épidémique}

\section{Mise en place d'une équipe de décision interdisciplinaire}

En dehors des situations de crise, les adaptations de l'offre hospitalière (régulation des lits et des services) et des plannings des équipes répondent à des règles administratives imposant de respecter des étapes de validations institutionnelles, nécessitant le plus souvent avant leur déploiement des délais de plusieurs semaines. L'évolution rapide de la crise sanitaire en France, les mesures de confinement national ainsi que la prévision à très courte échéance d'un pic épidémique en Lorraine ont conduit à mettre en place sur le site de la maternité un circuit décisionnel spécifique permettant une adaptation rapide tout en respectant le cadre réglementaire.

Une équipe de décision composée du chef de pôle de gynécologie-obstétrique, du responsable local du pôle d'anesthésie, du chef de service de pédiatrie, de la cadre supérieure de la maternité et du directeur référent a été mise en place. Son rôle était de coordonner de façon autonome et rapide l'organisation locale, de mettre en place des procédures spécifiques, de réguler et de transmettre les informations aux équipes. Ses actions étaient ensuite systématiquement relayées au niveau de la direction générale et de la commission médicale d'établissement.

\section{Arrêt des activités opératoires non urgentes}

Le 15 mars 2020, le Plan blanc a été activé au entre hospitalier régional universitaire (CHRU) de Nancy pour permettre l'accueil massif des patients victimes de la pandémie. Afin de répondre aux objectifs de limitation de la propagation virale au décours de soins non urgents, et de libérer des moyens humains et matériels vers les services déployés par le CHRU dans la lutte contre le SARS-CoV-2, les activités opératoires non urgentes ont été déprogrammées. Seules les activités urgentes et semi-urgentes définies initialement par l'équipe de décision ont été maintenues. Dans les semaines qui suivirent, des recommandations issues de la Société de chirurgie gynécologique et pelvienne et du Collège national des gynéco-obstétriciens français (CNGOF) ont définitivement validé les indications opératoires gynécologiques à maintenir à savoir les urgences infectieuses, hémorragiques et cancérologiques et l'activité réglée liées aux cancers, aspirations de grossesses arrêtées ou rétentions post-abortum et les ménométrorragies avec anémie après échec de traitement médical [1]. L'activité des interruptions volontaires de grossesses instrumentales a également été maintenue dans notre structure.

\section{Arrêt des activités de médecine de la reproduction}

Dès le 13 mars, l'Agence de biomédecine a confirmé l'arrêt des activités d'assistance médicale à la procréation. L'ensemble des actes hors préservations de fertilité pour cancer ont donc été suspendus. Seules les ponctions ovocytaires des patientes en fin de cycle de stimulation ont été maintenues jusqu'au 16 mars. Les dossiers de préservation de la fertilité ont été discutés au cas par cas en concertation clinicobiologique selon le degré d'urgence et les pathologies impliquées. Le maintien d'une astreinte téléphonique a été décidé pour répondre aux patientes en cours de traitement ou pour lesquelles un traitement était prévu. 


\section{Réorganisation des consultations}

Le 16 mars, l'ensemble des consultations externes a été réorganisé. Afin de limiter le risque de portage, un triage à l'entrée des consultations a été réalisé par des étudiants en médecine ou en maïeutique volontaires : interrogatoire à la recherche des signes classiques (fièvre, toux, diarrhées, exposition possible), prise de température, lavage de mains à la solution hydroalcoolique (SHA), rappel des règles de distanciation. Le port du masque en consultation et en échographie sur recommandations de la cellule d'hygiène n'était obligatoire que pour les personnels du CHRU du fait de stocks insuffisants, et d'une faible incidence du virus chez les patients asymptomatiques. À l'heure actuelle, le port du masque est obligatoire pour tous (patientes, accompagnants, personnels) dans l'établissement.

En gynécologie-obstétrique, les consultations ont été réorganisées par surspécialité. Après analyse en amont par les praticiens en charge des patientes, les consultations d'assistance médicale à la procréation et de gynécologie ont été limitées aux consultations semi-urgentes majoritairement en téléconsultation. En obstétrique, afin de respecter au mieux les recommandations de suivi des grossesses en fonction de leur niveau de risque ainsi que les prises en charge de médecine fotale, toutes les consultations ont également fait l'objet d'un triage en amont par les praticiens en charge des patientes afin de décider des modalités de réalisation des consultations (présentielles, téléconsultations ou suivi en ville). Une mobilisation des professionnels de ville (médecins généralistes, sages-femmes libérales) a été réalisée par le réseau périnatal lorrain et des procédures organisées pour le suivi des patientes à bas risque. Cependant, cette offre ayant été rapidement saturée pour réaliser les bilans cliniques (pression artérielle, bruits du cœur fœetal), et biologiques (chimie des urines, sérologies, hématobiologie) en externe, des consultations présentielles ont été largement maintenues. Les consultations urgentes de gynécologieobstétrique ont été rapidement réorganisées en deux filières distinctes dans l'espace : patientes non suspectes d'infection au SARS-CoV-2/patientes suspectes ou infectées. Le circuit « Covid-19» est décrit plus loin.

En anesthésie, les consultations de chirurgie réglée ont été annulées. Seules les consultations d'anesthésie pour des chirurgies gynécologiques semi-urgentes et l'activité obstétricale ont été maintenues soit par téléconsultation, soit par téléphone.

En pédiatrie postnéonatale, toutes les consultations présentielles ont été reportées à l'exception des consultations d'enfants en cours de traitement ou de suivi des nouveau-nés porteurs de pathologies à expression postnatale (ictères, suspicions d'infections secondaires tardives). Des téléconsultations par le néonatologiste habituellement en charge des enfants ont été mises en place afin de faire le point avec les parents sur le suivi des enfants et leur donner les informations disponibles quant au risque viral. Toutes les autres demandes ont été soit gérées par téléphone, soit orientées vers les urgences pédiatriques. Le centre de vaccinations dont la maternité a la charge a dans un premier temps été maintenu ouvert, en particulier pour les vaccinations BCG, en se limitant à un seul accompagnant. Celui-ci a ensuite été fermé lors du renforcement des mesures de confinement. Les nouveau-nés hospitalisés ont alors été vaccinés avant leur retour à domicile.

\section{Mesures générales dans l'établissement pour les soignants}

Tenant compte des recommandations émises par le service d'hygiène hospitalière du CHRU, et les sociétés savantes, l'état des connaissances, l'avis des équipes médicosoignantes locales, l'équipe de décision a mis en place en moins d'une semaine une série de mesures visant à limiter la propagation du virus au personnel soignant. Une formation de l'ensemble des personnels à l'habillage-déshabillage a été réalisée grâce à la diffusion du tutoriel YouTube de la Société française d'anesthésie et de réanimation ( SFAR ; https://sfar.org/covid-19/videos/) " habillage-déshabillage » et l'intervention de l'équipe opérationnelle d'hygiène de l'établissement. La dotation en matériels des différents services (SHA, lingettes désinfectantes, masques) a été coordonnée par les surveillantes de secteurs en lien avec la cellule d'hygiène en fonction des besoins mais surtout des stocks disponibles au plus fort de l'épidémie. Le port du masque chirurgical a été appliqué à tout le personnel soignant et/ou administratif en contact avec des patientes même asymptomatiques. Les tenues civiles ont été interdites, y compris pour les secrétaires en contact avec les patientes. Faute de masques, le port de masques à toutes les patientes n'a pu être réalisé. Un dépistage systématique des membres du personnel suspects a été proposé au niveau de l'unité de dépistage coordonnée par le service des maladies infectieuses. Les plannings de tous les personnels soignants et administratifs ont été redimensionnés afin de limiter la présence sur site aux personnels strictement nécessaires à l'activité non réalisable en télétravail. L'ensemble des stages de formation (externes, étudiants sages-femmes, infirmiers, élèves infirmiers anesthésistes) ont été reportés hormis pour les étudiants volontaires participant à l'évaluation du risque Covid19 aux différents points d'entrée du site et l'ensemble de leurs enseignements réalisés en ligne. La restauration des personnels s'est également adaptée, avec une fermeture du self du personnel et la distribution de repas à emporter.

\section{Mesures spécifiques par service}

En anesthésie, sur le plan des effectifs, du fait des tensions fortes au niveau des services de réanimation, les médecins 
extérieurs au service et assistants chefs de clinique réalisant des gardes dans le service ont été libérés de leurs obligations de garde pour aller renforcer les lignes de garde de réanimation. En effet, au plus fort de l'épidémie, le 4 avril, c'est plus de 200 lits de réanimation qui ont été ouverts contre 70 habituellement. Une liste d'astreinte pourvue par les médecins anesthésistes du site a été créée en plus de la garde habituelle afin de permettre la poursuite de l'activité classique en cas d'engagement du senior de garde sur un bloc avec une patiente Covid+ ou suspectée. Dans l'éventualité d'un absentéisme possible au sein de l'équipe comptant cinq médecins et d'une durée inconnue de la crise sanitaire, une liste de gardes d'anesthésistes seniors volontaires issus des établissements privés, ayant gardé une compétence en anesthésie obstétricale a été constituée. Celle-ci n'a finalement pas été engagée. Des procédures de prise en charge anesthésiques des patientes Covid + ou suspectes au bloc opératoire, en unité de soins continus, ont été réalisées ainsi qu'une veille bibliographique. Du fait de l'évolutivité des procédures mises en place, des connaissances sur le SARS-CoV-2 et des décisions institutionnelles en matière d'hygiène, une liste de diffusion via WhatsApp à tous les médecins et infirmiers anesthésistes du site a été créée afin de diffuser rapidement les informations essentielles, les messageries des uns et des autres étant surchargées. Les informations diffusées via WhatsApp relevaient uniquement des pratiques et matériels utilisés, aucune information confidentielle n'était diffusée par ce vecteur.

En gynécologie-obstétrique ont été désignés pour chaque type d'activité des binômes médecin-cadre responsables (obstétrique, gynécologie chirurgicale, gynécologie médicale). Afin de cloisonner au maximum le risque d'exposition et de transmission virale par et pour les soignants, une unité Covid-19 a été créée dès le 18 mars. Les durées de séjours des patientes hospitalisées, tous séjours confondus, ont été réduites au maximum et adaptées aux capacités de suivi posthospitalier des sages-femmes libérales, des médecins traitants ou de l'hospitalisation à domicile. Comme en consultation, le port du masque dans les services d'hospitalisation de gynécologie-obstétrique a été limité au personnel. Hors signes d'appel, aucun dépistage systématique du SARS$\mathrm{CoV}-2$ n'a été systématiquement réalisé en hospitalisation et à l'entrée en salle de naissance sur recommandations du service d'hygiène du fait de la faible incidence des patients asymptomatiques infectées au SARS-CoV-2, du manque de réactifs et de la tension importante existant au niveau du laboratoire de biologie. Le staff obstétrical journalier a été maintenu avec une présence minimale et un respect des règles de distanciation. Les réunions du centre de diagnostic prénatal ont été réalisées exclusivement en visioconférence.

En pédiatrie, chaque responsable d'unité fonctionnelle avec l'aide des cadres soignants a été chargé de proposer des procédures adaptées à son unité. Ces procédures rédigées à partir des recommandations émises par la Société française de néonatologie (SFN) dès le 10 mars et de la veille bibliographique mise en place dès le début de la crise sanitaire étaient ensuite validées collégialement. Un système de réunions dématérialisées par Microsoft Teams a été instauré dans le service afin de permettre des échanges instantanés et un point synthétique quotidien. Le port systématique du masque pour les soignants et les parents a été mis en place. En réanimation, la pression positive d'un tiers des boxes de soins de pédiatrie a été désactivée et remplacée par une pression négative. Ces boxes ont été spécifiquement réservés à la prise en charge des enfants nés de mères ayant un antécédent Covid + jusqu'à l'obtention des résultats de l'enfant. Un box d'accueil dédié à un isolement Covid+ a été créé en secteur de médecine néonatale pour la réalisation de photothérapies et le suivi de problèmes de puériculture ne pouvant pas être pris en charge au lit de la mère. Toutes les mamans du secteur Covid-19 ont reçu la recommandation de porter un masque systématiquement lorsqu'elles s'occupaient de leur enfant et de se laver les mains soigneusement. L'allaitement maternel direct a été tout particulièrement recommandé avec une procédure expliquée aux mamans. Cette procédure conseillait de se laver les mains, de mettre un masque tout au long de l'allaitement, puis de se désinfecter les mains au gel hydroalcoolique avant de nettoyer leurs seins selon les modalités habituelles. Conseil était donné de se désinfecter à nouveau les mains au gel hydroalcoolique avant de prendre l'enfant dans les bras et de se relaver les mains une fois l'allaitement terminé et l'enfant reposé dans son berceau.

\section{Accompagnants}

Le principe du confinement a impliqué de revoir radicalement les possibilités de visites au sein de la maternité. Un protocole de circulation des visiteurs dans l'établissement (salle de naissance, bloc opératoire, services, consultations) validé dans l'urgence par la direction et la commission d'éthique de l'hôpital puis confirmé par une note du CNGOF en date du 28 mars a été mis en place [2]. Dans les secteurs d'hospitalisation de gynécologie-obstétrique et notamment en suites de couches, aucune visite n'a été autorisée quel que soit le motif d'hospitalisation. En salle de naissance, la présence d'un accompagnant unique a été autorisée à l'accouchement ou en cas de complications majeures de la grossesse (fausses couches tardives, morts fœtales, pathologies fotales amenant à la réalisation d'une interruption médicale de grossesse) sous condition d'un examen clinique strictement normal (température, interrogatoire, paramètres vitaux), du port du masque et d'une protection vestimentaire (surblouse, charlotte). Au plus fort de l'épidémie, du fait de la configuration des locaux (absence de sanitaires individuels dans les boxes d'accouchement), cette présence a été limitée à la dernière phase du travail, afin de limiter les allers 
et venues des accompagnants au sein de la salle des naissances et de l'établissement. Toute sortie (tabac, toilette, alimentation) était considérée comme définitive. En cas de césarienne, aucune admission d'accompagnant au bloc opératoire n'a été admise. Néanmoins, un contact pèreenfant a été mis en place et réalisé par l'équipe de pédiatrie. Après les premiers soins, si l'état clinique de l'enfant était satisfaisant, un temps de peau-à-peau avec le papa était organisé. En néonatologie, seule la présence des parents a été autorisée en hospitalisation auprès de leur enfant. Dans les boxes multiples, les visites parentales ont été organisées selon un planning défini quotidiennement avec la soignante en charge de l'enfant par créneaux d'une heure avec un couple parental à la fois.

\section{Organisation de l'accueil et de la prise en charge des patientes suspectes ou infectées par la Covid-19}

\section{Urgences gynéco-obstétricales}

Comme en consultation, une zone de tri effectuée par un étudiant hospitalier a été créée à l'entrée du service des urgences (interrogatoire, prise de température, paramètres [saturation en air ambiant, fréquence cardiaque et respiratoire], lavage des mains et port du masque). En cas de doute sur une infection à SARS-CoV-2, la patiente était transférée dans l'unité de prise en charge Covid-19, après avis du médecin senior dédié à l'unité. Cette orientation était réalisée quel que soit le motif d'entrée, afin de créer un double circuit préservant le service des urgences classiques. Seules les patientes enceintes arrivant en phase expulsive étaient directement adressées en salle de naissance.

\section{Salle de naissance}

Une salle dédiée Covid-19 a été définie et armée du strict nécessaire en matériel obstétrical. Le dimensionnement des équipes a été adapté de sorte qu'une sage-femme puisse être systématiquement exclusivement dédiée à la prise en charge d'une patiente Covid + avec un habillage d'isolement contact et gouttelettes. En cas d'indication de césarienne en code rouge, les équipes d'anesthésie, d'obstétrique et de pédiatrie ont décidé en amont de façon consensuelle de respecter toutes les procédures d'hygiène et de sécurité nécessaires, malgré le retard potentiel qui en découlerait.

Sur le plan anesthésique, un chariot de matériels spécifiques à la pose d'une analgésie péridurale et/ou d'une rachianesthésie a été constitué. En cas de suspicion ou de cas avérés, les anesthésies locorégionales ont été exclusivement réalisées par les médecins seniors.
Le matériel pédiatrique de la salle de naissance a été adapté au risque de contamination virale (révision des respirateurs, mise en place de filtres de protection destinés aux respirateurs et au risque de diffusion externe via les circuits ouverts en l'absence de système en circuit fermé disponible en néonatologie). Le problème particulier des filtres inadaptés aux nouveau-nés et aux respirateurs spécifiques, générant une résistance du circuit et un espace mort excessifs, a été optimisé en utilisant le plus petit filtre pédiatrique disponible (filtre Humid Filter Pedia 11011T de Teleflex de $13 \mathrm{ml}$ d'espace mort) entre le cob et le spiromètre. La résistance a été compensée par une augmentation des pressions de ventilation de l'ordre de $2 \mathrm{cmH}_{2} \mathrm{O}$.

\section{Blocs opératoires gynécologiques et obstétricaux}

Sur le plan organisationnel, une salle de bloc a été dédiée à la prise en charge de toute patiente de gynécologie ou d'obstétrique suspecte ou confirmée d'infection à SARS-CoV-2. Dans cette salle, tout le matériel non indispensable a été retiré et les respirateurs équipés d'un second filtre en aval du tuyau d'expiration pour protéger le respirateur. Les systèmes de ventilation de la salle d'intervention n'ont pas été modifiés. Seules les substances les plus couramment utilisées pour réaliser une induction anesthésique et gérer les complications les plus courantes ont été laissées en dotation. Des « packs » préremplis ont été réalisés. En anesthésie, des kits succinylcholine et rocuronium, intubation difficile, rachianesthésie, péridurale, transfusion ont été réalisés. En gynéco-obstétrique, des kits césariennes, laparotomie, cœlioscopie, aspiration ont été préparés. Un chariot d'équipements pour les personnels a été disposé devant la salle. À l'entrée au bloc opératoire, toutes les patientes ont été équipées d'un masque chirurgical quelle que soit leur statut virologique [3].

Sur le plan de l'anesthésie, l'impossibilité de tester toutes les patientes admises au bloc opératoire lors du pic épidémique a contraint les équipes à uniformiser leurs prises en charge anesthésiques même chez les patientes asymptomatiques. L'anesthésie locorégionale comme préconisée par la SFAR a été systématiquement privilégiée et réalisée par un médecin senior expérimenté. En cas d'anesthésie générale, une préoxygénation au masque étanche avec branchement au circuit après application du masque était réalisée. L'intubation était systématiquement effectuée par un médecin senior ou un infirmier anesthésiste expérimenté, à l'aide d'un dispositif de vidéolaryngoscopie pour limiter au maximum l'exposition aux sécrétions. La sonde d'intubation était d'emblée reliée au filtre et au circuit pour limiter la transmission aérienne. Dans cette optique, l'absence de ventilation au masque était primordiale, ainsi que l'absence de toux. Une induction en séquence rapide était alors réalisée. Le branchement au respirateur était effectué une fois la pression du ballonnet monitorée. En cas de transfert d'une patiente intubée, 
le filtre patient était laissé sur la sonde d'intubation. L'extubation était réalisée au bloc opératoire, idéalement en présence uniquement de l'équipe d'anesthésie pour limiter le risque de contamination des autres soignants. Le débit de gaz frais était arrêté juste avant l'extubation une fois la patiente consciente, sans pression expiratoire positive. En cas de suspicion ou d'infection au SARS-CoV-2, la surveillance postinterventionnelle (SSPI) était réalisée en salle d'intervention puis la patiente adressée dans le secteur d'hospitalisation Covid+. En cas de signes de gravité préopératoire, la patiente était orientée vers l'unité de soins continus du service ou en réanimation si une extubation immédiate n'était pas possible. Pour toutes les anesthésies générales, l'équipe d'anesthésie était équipée de masque FFP2 et l'astreinte senior mobilisée. Les prescriptions d'anti-inflammatoires non stéroïdiens pour l'analgésie postopératoire et la prise en charge des douleurs pelviennes postaccouchement ont été suspendues du fait du risque d'aggravation d'une infection à SARS-CoV-2.

En gynéco-obstétrique, l'équipe était réduite au minimum à un médecin senior, un junior et une infirmière de bloc opératoire. L'astreinte senior était systématiquement appelée en renfort pour gérer l'activité habituelle.

En pédiatrie, un poste de travail spécifique a été organisé dans la salle Covid-19. Tout le matériel de protection et de prise en charge dédié était présent au niveau d'une table de réanimation installée sur place. Des kits spécifiques (détresse respiratoire, prise en charge hémodynamique) ont été préparés en plus du matériel habituel. En l'absence de signes de détresse, les enfants étaient mutés directement au niveau de l'unité Covid+ où ils étaient pris en charge par la sagefemme de l'unité en attendant leur mère.

\section{Unité de soins continus}

Un box vidé de tout le matériel non indispensable a été dédié à la prise en charge des patientes suspectées ou infectées à SARS-CoV-2. Du fait de l'occupation fréquente de tous les boxes de ce secteur en activité normale, le matériel et les équipements spécifiques Covid-19 pour le personnel ont été placés sur un chariot. La ventilation des chambres n'a pas été modifiée. Un protocole spécifique à l'unité a été rédigé afin d'uniformiser les prises en charge et le nettoyage. L'équipe soignante de cette unité étant composée d'une infirmière et d'une aide-soignante, une ligne d'astreinte de renfort infirmier mobilisable en cas de prise en charge d'une patiente suspectée ou infectée à SARS-CoV-2 a été créée afin de pourvoir assurer la continuité des soins des autres patientes présentes dans le service. De même, l'appel au médecin anesthésiste d'astreinte était prévu en cas de prise en charge mobilisant le médecin anesthésiste de garde (cathétérisme artériel, pose de voie veineuse centrale, intubation orotrachéale) chez une patiente à risque.

\section{Service d'hospitalisation Covid-19}

Un secteur d'hospitalisation spécifique Covid-19 a été créé dans un des secteurs de chirurgie libérés du fait de l'arrêt de l'activité opératoire. Ce secteur voué à l'accueil des urgences gynécologiques et obstétricales ainsi qu'aux hospitalisations des patientes suspectées ou infectées au SARS-CoV-2 était constitué de huit chambres d'hospitalisation classique, de deux salles d'examen, d'une salle de monitoring cardiofœtal, d'une salle d'échographie et de quatre salles d'attente isolées convertibles en lits d'hospitalisation (ramenant le capacitaire total d'hospitalisation à 12 lits). Un poste soignant disposant des matériels et des stocks de médicaments indispensables a été aménagé. L'équipement des chambres d'hospitalisation et des salles de consultation a été modifié. Tout le matériel non indispensable a été sorti, les arrivées d'oxygène vérifiées, et les équipements nécessaires (saturomètre, tensiomètre, thermomètre, matériel d'examen gynécologique, matériels de protection) mis à disposition sur une table devant chaque porte. Tout le matériel initialement utilisé dans les secteurs de soins fermés du fait de l'arrêt de l'activité chirurgicale a été réattribué au secteur Covid-19. Outre le matériel médical nécessaire au bon fonctionnement d'une hospitalisation conventionnelle, un échographe, plusieurs monitorings cardiofotaux, un chariot d'urgence adulte et pédiatrique dédiés ont été déployés au sein de l'unité. Le circuit de ventilation du service a été vérifié et adapté. En cas d'impossibilité d'instauration d'une pression de ventilation négative ou neutre, la ventilation communicante entre les pièces a été coupée et les chambres aérées plusieurs fois par jour. Le nettoyage des sols après chaque passage était effectué à l'aide d'un nettoyant désinfectant à spectre large pour sols et surfaces, après inutilisation de la salle pendant au moins 30 minutes. Un nettoyage biquotidien des parties communes du service a également été procédé selon ce même protocole de désinfection.

Concernant la gestion des effectifs soignants, un senior et un interne en gynéco-obstétrique, une sage-femme, une infirmière, un agent de service hospitalier ont été dédiés à l'unité. Une diffusion par e-mail au personnel médical de l'unité des protocoles de prise en charge régulièrement mis à jour a été organisée. Sur le plan de la formation des soignants aux mesures de protection, des séances répétées d'entraînements ont été réalisées concernant l'utilisation de surblouses à usage unique, surchaussures, masques chirurgicaux, charlottes, gants, lunettes de protection et l'utilisation efficace de SHA. Ces entraînements ont été d'autant plus importants que les pénuries de matériels ont contraint les équipes à s'adapter notamment lors des ruptures de surblouses en confectionnant de tenues en sacs-poubelle. Les masques FFP2 ont été réservés au personnel en cas de risque d'aérosolisation virale (prélèvement nasopharyngé, aspiration, aérosols, ventilation à haut débit...). 
Un parcours patient physique spécifique au secteur a été identifié dans l'établissement afin d'éviter les contacts avec des secteurs préservés. Toutes les patientes admises dans le secteur étaient accompagnées par un professionnel du service selon ce circuit spécifique. L'ascenseur menant au service a été isolé pour permettre le transport des patientes vers l'unité Covid-19, et les autres ascenseurs et accès de l'établissement menant au service Covid supprimés ou balisés. Dès leur arrivée dans le service, les patientes étaient immédiatement prises en charge et installées dans une pièce, adaptée aux soins prévus. Un relevé des paramètres vitaux et un monitoring cardiofotal pour les entrées obstétricales étaient réalisés ainsi qu'un prélèvement nasopharyngé à la recherche du coronavirus par PCR (polymerase chain reaction) chez toutes les patientes. Un examen clinique était effectué par un interne ou un senior de gynécologie obstétrique avec respect des mesures barrières et des consignes d'habillement et de protection individuelle. En cas de signes de gravité clinique (saturation $<90 \%$ en air ambiant ou oxygénothérapie à $3 \mathrm{l} / \mathrm{min}$, polypnée > 24 cycles/minutes, détresse respiratoire, mauvaise tolérance hémodynamique), un appel immédiat à l'anesthésiste réanimateur de garde était pris. À l'issue de cet examen, une décision de retour à domicile ou d'hospitalisation était retenue. Concernant les maturations fœtales en cas de risque d'accouchement prématuré, les indications habituelles de corticothérapies (quels que soient le terme et le statut Covid de la patiente) ont été maintenues aux vues des données de la littérature [4].

\section{Organisation du réseau périnatal et des transports dont le service d'aide médicale d'urgence (Samu)}

Comme les autres établissements de type 3, la maternité universitaire de Nancy, seul type 3 du réseau périnatal lorrain, est destinée à la prise en charge des femmes de son territoire de santé présentant une complication de leur grossesse avant 31 semaines d'aménorrhée (SA). Plusieurs maternités de type 2 à même de prendre en charge les complications obstétricales survenant avant 36 SA sont situées sur son territoire de santé, mais toutes ne disposent pas d'une réanimation. De fait, une procédure spécifique de transferts des femmes enceintes vers un établissement regroupant géographiquement une réanimation et une maternité de type adapté à l'âge gestationnel diffusé au Samu et à l'ensemble des établissements du réseau a été rédigée afin d'orienter les patientes vers l'établissement et le service le plus adapté. Afin de limiter le risque de naissances prématurées avant $31 \mathrm{SA}$ en maternité de type 1 ou 2 (outborn), une orientation des patientes oxygénodépendantes, vers la maternité régionale, a été recommandée du fait du risque d'aggravation brutale maternelle et de la morbi- mortalité plus importante des naissances prématurées hors établissement de type 3 [5].

\section{Difficultés rencontrées et pistes d'amélioration}

Si la plupart des actions de réorganisation mises en place ont donné entièrement satisfaction, les équipes ont tout de même rencontré plusieurs types de difficultés au plus fort de l'épidémie. Le volume d'activité et le nombre de patientes à risque d'être impliquées (suspicions d'infections ou infectées) dans ce contexte d'augmentation exponentielle des hospitalisations en services conventionnels et en réanimation ont été très difficiles à estimer car les retours d'expérience des autres pays (Chine, Italie) étaient alors quasiment inexistants. La mise en place précoce d'une concertation et de prises d'informations auprès des maternités alsaciennes touchées précocement a été un atout majeur permettant d'estimer l'impact de l'épidémie en obstétrique et de dimensionner les services, les personnels et les lits à ouvrir. Au début de l'épidémie, les publications en obstétrique étaient extrêmement peu nombreuses, rendant d'autant plus difficile l'anticipation des prises en charge. La mise en place d'une veille bibliographique est un élément indispensable à mettre en place.

La transmission efficace de l'information entre personnel médical et paramédical a été un réel défi. La rapidité d'évolution de l'épidémie, des connaissances, des procédures a été une difficulté importante. L'organisation de réunions de crise hebdomadaires, voire bihebdomadaires dématérialisées, a permis un relais de l'information plus performant que la communication initiale par e-mails. La création de groupes WhatsApp pour la diffusion d'informations purement structurelles et matérielles a également permis de favoriser l'information dans les équipes.

Des problèmes éthiques comme le maintien ou non de la présence d'un accompagnant en salle de naissance sont rapidement devenus essentiels. Dans l'urgence, l'aide du conseil d'éthique de l'établissement a permis aux équipes de réfléchir et de déterminer la meilleure décision à prendre. Les différentes sociétés savantes nationales (CNGOF, SFAR, $\mathrm{SFN}$ ) et internationales ainsi que les autorités sanitaires nationales ont rapidement émis des procédures et recommandations permettant pour certaines de valider les décisions prises en urgence sur le terrain comme le choix des interventions à maintenir, la présence des accompagnants. Les recommandations en matière d'hygiène (équipements, habillage) par contre se sont rapidement heurtées aux pénuries d'approvisionnement, et au manque de stocks, générant des tensions et incompréhensions entre équipes soignantes et équipes d'hygiène. En effet, au plus fort de l'épidémie, les procédures en matière d'hygiène notamment en termes de protections individuelles ont été réalisées en fonction des stocks de matériels disponibles et de la prévalence de la 
maladie chez les patientes asymptomatiques et non en vertu des recommandations des sociétés savantes. Ces divergences entre recommandations nationales et locales ont été source d'angoisse pour le personnel craignant d'être insuffisamment protégé contre le virus. Une communication franche et éclairée des équipes d'hygiène avec les équipes soignantes est indispensable.

En conséquence des pénuries, le port du masque chirurgical pour toutes les entrées (consultations, hospitalisation) n'a ainsi pas pu être mis en place, seules les patientes suspectées ou infectées adressées en secteur Covid+ en ont bénéficié. De même, un dépistage large de toutes les patientes admises en hospitalisation ou en salle de travail n'a pas non plus pu être organisé du fait d'une pénurie de réactifs de biologie et d'une saturation du service de biologie. Cela a conduit l'équipe d'anesthésie à s'interroger quant à la prise en charge des patientes asymptomatiques nécessitant une chirurgie semi-urgente sous anesthésie générale. De fait, toutes ont été prises en charge comme des patientes suspectes (crush induction, réveil en salle d'intervention), ce qui n'aurait pas été le cas en cas de dépistage. Pour la même raison, chaque salle d'intervention a été réorganisée de façon à limiter le matériel présent au strict nécessaire d'autant plus qu'une patiente asymptomatique à son entrée, opérée d'une grossesse extra-utérine, s'est révélée positive en postopératoire lors d'un bilan d'hyperthermie postopératoire. Les préconisations de limitation des personnels présents notamment lors du réveil postopératoire (réveil agité), l'installation des urgences gynécologiques ou obstétricales extrêmes (césarienne code rouge, chocs hémorragiques, grossesse extrautérine rompue...) peuvent mettre les équipes en difficulté et nécessite de disposer d'un renfort en personnels immédiatement mobilisable.

$\mathrm{Du}$ fait du risque de contamination des personnels, et d'une focalisation de l'attention sur le SARS-CoV-2, un impact direct sur les prises en charge habituelles de pathologies gynécologie-obstétricales a été relevé tout particulièrement en cas d'hyperthermie maternelle avec des retards de mise en place d'antibiothérapie. Un rappel régulier aux équipes locales et régionales par l'intermédiaire du réseau périnatal lorrain est à entreprendre afin d'assurer une prise en charge des patientes selon les règles de bonnes pratiques habituelles.

Le problème de vol du matériel (masques, SHA) s'est révélé problématique du fait des stocks limités et la disponibilité des équipements de protection pour les équipes. Restreindre les points d'accès aux masques et au matériel de protection, au gel hydroalcoolique placé dans les armoires à pharmacie est vite apparu nécessaire. Les contenants de poche de gel hydroalcoolique ont été remplacés par des conditionnements plus volumineux plus difficiles à dissimuler.

\section{Discussion}

La littérature actuelle concernant l'organisation à mettre en place en maternité face à la survenue d'une crise sanitaire de type pandémie est très limitée. En situation de pandémie, l'adaptation rapide des structures hospitalières doit répondre à un double défi : participer au confinement de la population dans l'objectif de limiter la propagation virale et de répondre aux impératifs spécifiques de prise en charge de l'ensemble des patientes qu'elles soient ou non infectées. Face à l'évolutivité extrêmement rapide de la situation dans la région Grand Est, un retour d'expérience de l'organisation mise en place dans les premières maternités touchées nous paraissait important afin de souligner non seulement les actions réalisées, mais également celles qui auraient dû être réalisées et les difficultés rencontrées. Le but de cet article était de servir de base de réflexion aux maternités, quel que soit leur niveau de prise en charge, confrontées encore actuellement au SARS-CoV-2 et de proposer un modèle transposable à d'autres pathologies épidémiques.

À ce jour, l'impact du virus Covid-19 sur les femmes enceintes n'a ni plus ni moins suivi celui de la population générale avec une incidence des décompensations respiratoires sévères dans un ratio similaire [4,6-9]. Par rapprochement avec les autres étiologies virales, les complications infectieuses respiratoires sont pour autant une cause importante de morbimortalité périnatale en termes de risques de naissances prématurées, de morts fœtales in utero, de retards de croissance intra-utérins et de décès néonataux [10-14]. Les séries de cas rapportés actuellement ne semblent pas confirmer cette tendance [4,15-17], mais ces premiers rapports sont très préliminaires, et de nombreux centres français ont alerté quant à la survenue de complications sévères chez des femmes enceintes en lien avec le SARS-CoV-2. Au 30 mars, notre équipe avait déjà pris en charge sept femmes enceintes présentant une détresse respiratoire sévère justifiant d'une oxygénothérapie, compliquées de deux naissances prématurées induites dans un contexte de détresse respiratoire aiguë avec intubation maternelle. Au total, du 18 mars 2020 au 11 mai 2020, 67 hospitalisations ont eu lieu dans le service Covid de la maternité de Nancy.

La mise en place rapide de zones de triage en consultations et aux urgences permettant de discerner deux circuits de soins, la formation des équipes en amont de la survenue de l'épidémie (habillage, mesures de prévention) validées lors d'autres épidémies, comme Ebola notamment, ont permis de limiter la propagation virale dans l'établissement $[16,18,19]$.

Une évaluation des ressources humaines nécessaires au fonctionnement de la structure (bloc opératoire, soins continus, consultations, pédiatrie) est un élément crucial de l'organisation des soins en situation de crise sanitaire afin de 
mobiliser les ressources vers d'autres secteurs ou activités. Ainsi, les chefs de cliniques du service d'anesthésie ont renforcé les réanimations, les infirmières anesthésistes venues en renfort du secrétariat d'anesthésie ont permis en moins d'une semaine de réorganiser complètement les consultations du service. Des étudiants hospitaliers et en maïeutique ont assuré les points de triage. Les internes de gynécoobstétrique et d'anesthésie-réanimation ont assuré la veille bibliographique. Une aide spontanée des médecins anesthésistes-réanimateurs libéraux partiellement libérés de leurs obligations avait également été proposée. En cas de prolongation de l'épidémie, cette aide aurait été sollicitée.

Le maintien de l'activité de consultations obstétricales utile du fait de la saturation des dispositifs de ville et la mise en place rapide de téléconsultations ont permis de poursuivre le suivi des patientes et de limiter stress et anxiété. Le risque de transmission à leur enfant, le confinement, les sujets contacts, l'éloignement familial, la présence du conjoint à l'accouchement ont été nombre de sources de stress à lever. Or, l'association stress et grossesse est connu pour générer de la pathologie obstétricale, d'où l'importance de maintenir le lien avec les patientes qui pourraient aussi ne pas continuer un suivi régulier de peur de venir à l'hôpital, jugé lieu de contamination possible. La mobilisation rapide du Club des anesthésistes réanimateurs en obstétrique a permis également en plus de la mise en place des téléconsultations via le lien « préanesthesie » de fournir un support rassurant pour les patientes, lien largement diffusé à chaque patiente en amont des consultations.

\section{Conclusion}

L'arrivée précoce de la pandémie à SARS-CoV-2 en région Grand Est s'est révélée pour tous les établissements hospitaliers un véritable défi en matière d'organisation et d'adaptation. Dans les maternités, la poursuite des activités de suivi et de prise en charge a nécessité de garantir aux patientes et à leurs enfants une qualité des soins constante tout en minimisant le risque de contamination virale. Le triage des patientes, l'organisation de circuits spécifiques, la mise en place de téléconsultations et la veille bibliographique instaurée ont permis de garantir au mieux la sécurité des patientes et des personnels.

Remerciements : les auteurs remercient le Pr Philippe Deruelle pour son aide précieuse lors de la rédaction de cet article.

Liens d'intérêts : les auteurs déclarent ne pas avoir de liens d'intérêts.

\section{Éléments remarquables}

- La mise en place d'une équipe de décision a facilité la coordination autonome et rapide de l'organisation locale et des procédures, et de transmettre les informations aux équipes - L'arrêt des activités opératoires et des consultations non urgentes a permis de libérer personnels, matériels et locaux - Les décisions concernant les indications opératoires à poursuivre, la gestion des accompagnants ont été prises précocement avec l'aide du comité d'éthique d'établissement - Le triage des entrées (consultations, hospitalisations et urgences) a été un atout important de limitation de la propagation virale - Un renforcement des équipes en cas de prise en charge en urgence (bloc, soins continus en salle de naissances) de patientes Covid+ a permis la poursuite des activités urgentes classiques

- La création d'un service Covid+ avec un parcours patient spécifique est indispensable pour limiter la propagation du virus, de faciliter les prises en charge médicales, la formation des personnels et les dotations de matériel

- L'orientation des patientes oxygénodépendantes vers l'établissement de type 3 régional a permis de limiter la morbimortalité des naissances prématurées

\section{Pistes d'amélioration}

- La prise d'information auprès des premières maternités touchées et la mise en place d'une veille bibliographique précocement sont des atouts majeurs permettant d'estimer l'impact l'épidémie en obstétrique et de dimensionner les services, les personnels et les lits à ouvrir - L'organisation de réunions de crise hebdomadaires, voire bihebdomadaire et la création de groupes WhatsApp pour la diffusion d'informations structurelles et matérielles sont des outils d'information aux équipes plus performants que les messageries électroniques

- Une communication franche et éclairée des équipes d'hygiène avec les équipes soignantes est indispensable

- Un dépistage systématiquement des patients admis au bloc opératoire pour une chirurgie réglée permet de limiter les prises en charge de Covid+ aux seules patientes suspectes (équipement des équipes, crush induction, réveil en salle d'intervention) - Les réseaux périnataux doivent jouer un rôle important en termes d'organisation des soins et de transferts de patientes 


\section{Références}

1. Akladios C, Azais H, Ballester M, et al (2020) Recommendations for the surgical management of gynecological cancers during the Covid-19 pandemic - FRANCOGYN group for the CNGOF. J Gynecol Obstet Hum Reprod 49:101729

2. Peyronnet V, Sibiude J, Deruelle P, et al (2020) Infection par le SARS-CoV-2 chez les femmes enceintes. État des connaissances et proposition de prise en charge. CNGOF. Gynecol Obstet Fertil Senol 48:436-43

3. Société française d'anesthésie et de réanimation (2020) Aide cognitive - prise en charge en anesthésie-réanimation d'une patiente Covid+ ou suspecte en maternité. https://sfar.org/download/aide-cognitive-prise-en-charge-ar-patiente-covid-ou-suspecteen-maternite/ (Dernier accès le 10 juillet 2020)

4. Chen D, Yang H, Cao Y, et al (2020) Expert consensus for managing pregnant women and neonates born to mothers with suspected or confirmed novel coronavirus (Covid-19) infection. Int J Gynecol Obstet 149:130-6

5. Hossain S, Shah PS, Ye XY, et al (2016) Outborns or inborns: where are the differences? A comparison study of very preterm neonatal intensive care unit infants cared for in Australia and New Zealand and in Canada. Neonatology 109:76-84

6. Fan C, Lei D, Fang C, et al (2020) Perinatal transmission of Covid-19 associated SARS-CoV-2: should we worry? Clin Infect Dis 71 (in press)

7. Qiao J (2020) What are the risks of Covid-19 infection in pregnant women? Lancet 395:760-2

8. Jiao J (2020) Under the epidemic situation of Covid-19, should special attention to pregnant women be given? J Med Virol 92:1371-2

9. Schwartz DA (2020) An analysis of 38 pregnant women with Covid-19, their newborn infants, and maternal-fetal transmission of SARS-CoV-2: maternal coronavirus infections and pregnancy outcomes. Arch Pathol Lab Med 145 (in press)

10. Schwartz DA, Graham AL (2020) Potential maternal and infant outcomes from coronavirus 2019-nCoV (SARS-CoV-2) infecting pregnant women: lessons from SARS, MERS, and other human coronavirus infections. Viruses 12:194

11. Benedetti TJ, Valle R, Ledger WJ (1982) Antepartum pneumonia in pregnancy. Am J Obstet Gynecol 144:413-7

12. Berkowitz K, LaSala A (1990) Risk factors associated with the increasing prevalence of pneumonia during pregnancy. Am J Obstet Gynecol 163:981-5

13. Rigby FB, Pastorek JG (1996) Pneumonia during pregnancy. Clin Obstet Gynecol 39:107-19

14. Madinger NE, Greenspoon JS, Gray Ellrodt A (1989) Pneumonia during pregnancy: has modern technology improved maternal and fetal outcome? Am J Obstet Gynecol 161:657-62

15. Rasmussen SA, Smulian JC, Lednicky JA, et al (2020) Coronavirus disease 2019 (Covid-19) and pregnancy: what obstetricians need to know. Am J Obstet Gynecol 222:415-26

16. Chen Y, Li Z, Zhang YY, et al (2020) Maternal health care management during the outbreak of coronavirus disease 2019 (Covid19). J Med Virol 92:731-9

17. Liu D, Li L, Wu X, et al (2020) Pregnancy and perinatal outcomes of women with coronavirus disease (Covid-19) pneumonia: a preliminary analysis. Am J Roentgenol 215:127-32

18. Liang H, Acharya G (2020) Novel corona virus disease (Covid19) in pregnancy: what clinical recommendations to follow? Acta Obstet Gynecol Scand 99:439-42

19. Quaglio G, Pizzol D, Bome D, et al (2016) Maintaining maternal and child health services during the Ebola outbreak: experience from Pujehun, Sierra Leone. PLoS Curr 8: ecurents.outbreaks. d67aea257f572201f835772d7f188ba5 